\title{
Du théâtre à l'amphithéâtre : pour une extension du concept d'adhésion à la neuroscience éducationnelle
}

https://doi.org/10.24046/neuroed.20130201.1

\author{
Safouane M. HAMDI ${ }^{1, ~}{ }^{*}$ et Yannick BRESSAN ${ }^{2}$
}

1 Université de Toulouse

2 Université de Strasbourg

* courriel : safouane.hamdi@univ-tlse3.fr

\begin{abstract}
Résumé
Une expérience interdisciplinaire, réalisée en 2007 à Strasbourg (France) a permis de déterminer les corrélats neuronaux et physiologiques du principe d'adhésion à une réalité fictive (théâtrale). Elle a démontré que l'adhésion à cette réalité fictionnelle est induite par une mise en scène spécifique. Une analyse phénoménologique des liens entre le théâtre et l'enseignement a permis de poser l'hypothèse que l'adhésion est également mise en œuvre en situation pédagogique et qu'elle impacte l'apprentissage. Il est en effet admis que les enseignants tentent de construire, consciemment ou non, une représentation $d u$ réel pour favoriser l'apprentissage des étudiants. Les moyens à l'œuvre relèvent donc d'une "mise en scène pédagogique ». Le présent article pose les bases théoriques de l'adhésion dans le champ pédagogique et propose un cadre expérimental ancré dans la neuroscience éducationnelle. En effet, les situations d'apprentissage étant complexes, seule une combinaison de méthodologies permet une meilleure compréhension des processus cognitifs sous-jacents. Ainsi, pour démontrer l'adhésion des étudiants au cours d'une intervention éducative, plusieurs conditions sont nécessaires: l'intention de l'enseignant véhiculée par la mise en scène pédagogique, la convergence de corrélats physiologiques et neurologiques spécifiques et la confirmation subjective des étudiants eux-mêmes. Sur cette base, deux expériences pilotes ont été conçues pour répondre aux questions suivantes: est-il possible d'induire l'adhésion des étudiants à un cours magistral ? Le concept connexe de mise en scène pédagogique améliorerait-il leur mémorisation des informations transmises ainsi que leurs résultats aux tests d'évaluation par rapport à une transmission plus classique des savoirs ?
\end{abstract}




\section{Introduction}

Le théâtre est défini comme un art dont le but est de donner à voir et à entendre à un public, une suite d'évènements et d'actions par le biais d'acteurs qui se déplacent sur une scène et qui utilisent le discours et l'expression corporelle. II représente donc une forme de réalité circonscrite dans l'espace et le temps. À travers l'histoire, les metteurs en scène ont cherché, par l'écriture et la mise en scène, à faire croire à cette réalité fictionnelle. Le paradoxe est que le spectateur entre dans cette réalité, il y adhère en laissant momentanément de côté la réalité physique et sensorielle qui l'entoure. Quels sont donc les processus cognitifs, neurologiques et physiologiques qui conduisent un spectateur à percevoir Hamlet à la cour d'Elseneur alors qu'il a sous les yeux un comédien sur des planches de théâtre?

Une expérience interdisciplinaire a permis de déterminer les corrélats neuronaux et physiologiques de cette adhésion du sujet-spectateur (Bressan, 2012; Metz-Lutz, Bressan, Heider et Otzenberg, 2010). Pour cette expérience, un dispositif théâtral a été recréé en laboratoire (mise au noir, lumières, etc.). Des représentations d'Onysos le Furieux (Gaudé, 2000) ont été jouées par un comédien professionnel, filmées et diffusées en direct à 20 sujets-spectateurs. Le metteur en scène, lors de son travail préparatif de la représentation, dirigeait le comédien en lui demandant de souligner certains moments de la représentation par de subtiles attitudes corporelles, des regards, un rythme de voix ou des déplacements particuliers. Les expérimentateurs ont précisément identifié ces interventions en les nommant « événements-théâtre ». Durant les 14 minutes de la représentation, ils ont acquis des images IRMf et enregistré un électrocardiogramme pour chacun des sujets-spectateurs. Dans une approche neurophénoménologique, ils ont complété l'acquisition des données neurologiques et physiologiques par une «méthodologie à la première personne " en faisant remplir aux sujets un questionnaire a posteriori. Chacun d'eux a commenté ses impressions tout au long de l'expérience et a indiqué les moments où il a adhéré à la réalité fictionnelle (événements subjectifs). De manière intéressante, les évènements-théâtre préalablement définis par le metteur en scène ont coïncidé dans $69 \%$ des cas avec les évènements subjectifs et dans $80 \%$ des cas, avec des modifications de l'activité cérébrale. Parmi les régions cérébrales dont l'activité a été significativement modifiée par les évènements-théâtre (par comparaison à des moments neutres), les expérimentateurs ont identifié la zone temporo-pariétale gauche, le gyrus frontal inférieur, le sillon temporal supérieur gauche et le précuneus. Ils ont également repéré une diminution de la variabilité du rythme cardiaque durant ces évènements. L'ensemble des résultats ont amené les expérimentateurs à discuter l'hypothèse selon laquelle les évènements-théâtre font non seulement 
intervenir les zones cérébrales impliquées dans l'empathie, la métaphore et la théorie de l'esprit mais qu'ils induisent aussi un changement subtil de l'état de conscience des spectateurs. Ce changement les a amenés à une perte temporaire de la sensation du corps et de la représentation du soi. L'adhésion d'un sujet à une réalité théâtrale procède donc d'un "basculement ", c'est-àdire d'une dissociation entre une expérience mentale en cours et l'expérience physique immédiate. Au cours de l'adhésion, le sujet libère son esprit et son attention de ses sensations immédiatement perceptibles afin d'accéder à la fiction dramatique. Ce phénomène est borné dans l'échelle du temps, il est sous-tendu par des processus physiologiques et cognitifs définis et il est identifiable a posteriori par le sujet lui-même. Marie-Noëlle Metz-Lutz, qui a contribué à l'étude, commente ainsi ces résultats (Bressan, 2013, p. 149) :

" L'adhésion est inhérente au théâtre même, puisque ce que va en

faire le metteur en scène, c'est chercher à faire adhérer le spectateur, et le spectateur se laisse tromper; il vient pour être trompé. Le théâtre est peut-être la situation expérimentale idéale où on introduit dans la perception du fait une perception faussée, décidée, mise en scène. C'est le principe de tout discours: introduire dans la pensée de l'autre un déplacement de perception. "

Cette première étude a permis de déterminer les corrélats neurologiques et physiologiques de l'adhésion du sujet-spectateur à la réalité théâtrale mais elle met aussi en relief deux autres notions. D'abord, le metteur en scène, présumant de l'adhésion du spectateur, détiendrait ainsi un pouvoir de manipulation sur le sujet. Ce pouvoir est cependant atténué par la diversité des spectateurs qui ne percevraient pas tous les évènements-théâtre de la même manière. Ensuite, le théâtre apparaît comme un cadre expérimental particulièrement riche pour les sciences cognitives (Bressan, 2012). C'est d'ailleurs à ce titre qu'ont émergé les premières questions à propos de l'extension du concept d'adhésion au champ pédagogique. Les proximités observables entre les cadres théâtral et éducationnel peuvent-elles conduire à concevoir une "mise en scène pédagogique "? Si oui, celle-ci pourrait-elle venir renforcer la transmission des savoirs?

\section{Le cadre théorique: extension du concept d'adhésion au champ pédagogique}

Les rapports entre le théâtre et la pédagogie sont anciens mais ambigus et le Cours Magistral (CM), exercice emblématique de l'enseignement universitaire, en est une des meilleures preuves. En effet, la théâtralité du CM n'est pas une simple métaphore suggérée par la disposition des lieux où il est donné (amphithéâtre en français, lecture theater en anglais), elle est ontologique ce qui implique que, par essence et depuis ses origines, le CM est un exercice 
théâtral. Un bel exemple vient de la description précise des cours du philosophe Hegel rapportée en 1835 par un de ses auditeurs, Hotho, et récemment commentée par Pierre Macherey (2011) :

Le premier enseignement qui se dégage de cet impressionnant morceau d'écriture, dont l'auteur s'est employé à restituer, au plus près de ce qu'il avait éprouvé sur le moment, son expérience personnelle d'auditeur des cours de Hegel, c'est que, tels que ce dernier en gérait l'économie, ils étaient montés comme un spectacle qui, en mettant en scène la parole professorale, exploite tous les ressorts d'une action dramatique. Hegel ne se contentait pas d'exposer en chaire des idées, en les alignant platement et machinalement les unes à la suite des autres, comme si elles coulaient de source; mais, avec les moyens du théâtre, il donnait la démonstration d'un " effort », pour reprendre le terme qui revient tout au long du témoignage de Hotho et en constitue le fil conducteur.

Les recherches historiographiques ont montré que cette théâtralité a été initialement reconnue et valorisée par l'Université puis progressivement mise au second plan pour des raisons didactiques (Noguès, 2008). Les approches pédagogiques "actives » et centrées sur l'étudiant, apparues vers la fin du $\mathrm{XXe}$ siècle, ont sévèrement critiqué le $\mathrm{CM}$ et son côté théâtral, jugé comme une tradition archaïque et contre-productive (Charles, 1997). Dès lors, la théâtralité du CM est rejetée dans un subconscient universitaire collectif, mais elle réapparaît régulièrement dans la littérature pédagogique comme un élément métaphorique et non plus ontologique. Ainsi, plusieurs auteurs ont appelé, à intervalles réguliers, les enseignants à s'inspirer de l'acteur de théâtre pour innover, améliorer ou s'approprier leurs propres pratiques (Harris, 1977; Murray et Lawrence, 1980; Waack, 1983; Greenberg et Miller, 1991; Pineau, 1994; Griggs, 2001; Baruch, 2006). La théâtralisation de l'enseignement permettrait également aux éducateurs de s'affirmer, de gagner en confiance et de mieux gérer la diversité de l'auditoire (Feagan et Rossiter, 2011; Souto-Manning, 2011). Cette approche métaphorique est, certes, féconde mais elle permet de contourner avantageusement les craintes et/ou le refus des enseignants d'êtres considérés comme des acteurs, éludant ainsi un intéressant débat de fond. Une autre façon de rapprocher théâtre et enseignement est de considérer que les deux contribuent à créer une réalité ou, du moins, une certaine représentation de la réalité avec laquelle les spectateurs/étudiants sont invités à interagir.

La proximité entre le théâtre et la pédagogie est donc patente, mais elle est complexe dans le sens où sa perception et son usage ont évolué dans le temps. Néanmoins, elle autorise un transfert analogique du concept d'adhésion 
décrit ci-dessus dans le champ éducatif. Intuitivement, le pédagogue qui s'intéresse au sujet va avoir une double interrogation: les étudiants en situation d'auditeurs d'un cours magistral peuvent-ils adhérer au discours de l'enseignant? De quels ressorts ce dernier use-t-il pour susciter l'adhésion des étudiants? Là aussi, une analyse de la littérature montre que ce concept a déjà été pressenti dans le champ pédagogique sur des bases intuitives ou phénoménologiques parfois anciennes ou plus récemment au travers de la notion d'intérêt situationnel (Schraw et Lehman, 2001; Hidi et Renninger, 2006; Dohn, Madsen et Malte 2009). Un nouveau champ de recherches s'ouvre donc. En s'appuyant sur la neuroéducation comme paradigme de référence pour délimiter les cadres théorique et expérimental, les premières questions de recherche qui émergent sont les suivantes: est-il possible d'induire l'adhésion des étudiants lors d'un cours magistral? L'adhésion a-t-elle un impact sur leurs apprentissages? Quels en sont les ressorts cognitifs et neurologiques? Peut-elle être mise en œuvre dans d'autres situations pédagogiques (e-learning)?

Ces premières questions de recherche vont guider les outils expérimentaux à mettre en œuvre dont certains seront proposés plus loin. Elles suscitent également des développements théoriques intéressants au sujet de l'impact potentiel de l'adhésion sur les deux protagonistes du CM: les étudiantsspectateurs et l'enseignant-acteur. Concernant les étudiants, l'adhésion pourrait interférer avec leurs apprentissages de plusieurs façons : élévation de leur niveau d'attention, augmentation de l'intérêt situationnel, amélioration des processus de mémorisation et diminution de la charge cognitive durant les CM. Concernant l'enseignant, s'il recherche consciemment ou non l'adhésion des étudiants, il devra adapter par divers moyens sa conduite du CM. II pourra, entre autres, retravailler son message pédagogique sur le fond et sur la forme, adapter sa voix et sa gestuelle. De manière intéressante et comme nous l'avons déjà souligné, la littérature traite de ces adaptations en les désignant comme de simples "recettes " ou tips inspirées du théâtre. Ainsi, nous proposons que le concept d'adhésion soit d'abord subordonné à une intention de l'enseignant puis à la mise en place par ce dernier d'un ensemble d'actions regroupées dans une " mise en scène pédagogique » (Bressan, 2012b). Enfin, il est tout à fait envisageable que d'autres facteurs interviennent dans l'adhésion des étudiants comme la taille du groupe et ses interactions ou l'interposition d'un écran entre l'enseignant et les étudiants.

Outre leur apport purement conceptuel, ces développements théoriques permettent de guider les approches expérimentales en identifiant les variables qu'il est possible de modifier pour explorer l'adhésion en tant que phénomène cognitif dans une situation pédagogique. 


\section{Le cadre expérimental pour explorer le concept d'adhésion dans le champ pédagogique}

La mise en évidence du concept d'adhésion dans le contexte théâtral a été réalisée par une double approche, subjective et objective. Une approche similaire devrait donc être utilisée pour son exploration dans un contexte pédagogique. Or, la recherche en neuroscience éducationnelle s'organise autour d'un consensus méthodologique (Campbell et Pagé, 2012; Mercier, Léger, Girard et Dion, 2012) qui allie les méthodes dites à la troisième personne (recueil de données psychophysiologiques) et celles dites à la première personne (analyse du comportement, du discours, etc.). II apparaît ainsi que le cadre expérimental à mettre en œuvre pour explorer l'adhésion en pédagogie s'ancre naturellement dans le champ de la neuroéducation. La nouveauté est conceptuelle mais les jalons expérimentaux sont déjà posés (Metz-Lutz et al., 2010; Campbell et Pagé, 2012).

La principale hypothèse que nous souhaitons tester est la suivante: "Est-il possible d'induire l'adhésion des étudiants au cours d'une intervention éducative? ". Les expériences qui seront construites en ce sens reposeront sur deux éléments: une intervention éducative spécifique et un recueil de plusieurs types de données. Le protocole devra être soumis et approuvé par le comité de protection des personnes de l'institut de recherche.

\subsection{L'intervention éducative}

L'intervention consiste en un cours magistral délivré par un enseignant expérimenté. II a été montré que l'adhésion à une réalité fictionnelle est très dépendante de l'intention du metteur en scène (Bressan, 2012; Metz-Lutz et al., 2010). II convient donc que le $\mathrm{CM}$ intègre de manière claire et repérable l'intention de l'éducateur de faire adhérer les étudiants. Ce sera le cas au travers de la mise en scène pédagogique du CM. L'enseignant présentera certains messages pédagogiques dont le contenu et la forme seront spécifiques et préparés en amont. En se basant sur l'étude de Metz-Lutz et al. (2010), nous postulons que ces messages pédagogiques mis en relief, ou mis en scène, susciteront l'adhésion des étudiants.

\subsubsection{Les participants}

Les participants à l'expérience seront des étudiants adultes en $2^{\mathrm{e}}$ cycle d'études de santé (médecine, pharmacie, élèves sages-femmes) ayant déjà une bonne expérience du CM. L'effectif sera de 12 étudiants $(6$ hommes, 6 femmes) réputés indemnes de pathologie neurologique ou cardiaque. Ils ne 
seront pas rémunérés, mais recevront une version écrite du $\mathrm{CM}$ un mois après l'expérience.

\subsubsection{Le CM}

Le cours a pour intitulé: "Modifications métaboliques et hématologiques au cours de la grossesse ». II développe certaines des modifications physiologiques de l'organisme d'une femme enceinte. Le sujet a été choisi en raison de l'intérêt potentiel qu'il peut susciter chez les étudiants. En effet, tout futur professionnel de santé devrait connaître ces notions, mais elles sont rarement abordées dans les cursus académiques généralistes. Le CM a une durée de 20 minutes et sera délivré en matinée. II se décline en 18 diapositives projetées sur un écran. La première diapositive présente le titre du cours, chacune des 17 suivantes délivre ensuite un message pédagogique unique. Les messages sont décrits succinctement dans le Tableau 1.

\subsubsection{La mise en scène}

La mise en scène pédagogique révèle l'intention de l'enseignant. Elle est, par postulat, indispensable à l'adhésion des étudiants. Étant un concept nouveau et non théorisé, nous en posons les premières bases selon une approche phénoménologique, à la lumière de notre expérience pédagogique et scénique. Par analogie avec l'importance du texte et de l'acteur dans la représentation théâtrale, nous proposons une première typologie des mises en scène pédagogique basée sur la place de l'enseignant et du support visuel dans le CM. D'autres éléments peuvent intervenir, mais pour ces premières expériences, nous avons élaboré deux mises en scène diamétralement opposées. Le choix de cet antagonisme repose sur l'hypothèse que tous les étudiants seront sensibles à l'une au moins de ces méthodes :

- Méthode 1 ou « avec acteur et sans support»: l'enseignant prend le pas sur le support. II projette une diapositive noire, attire l'attention des étudiants vers lui, les interpelle sur l'importance du message pédagogique et le délivre avec une gestuelle et une intonation vocale qui soulignent cette importance.

- Méthode 2 ou " avec support et voix off »: l'enseignant s'efface au profit du support, il n'intervient que par la voix et utilise un mode narratif. Dans ce CM, la diapositive projette le fac-similé d'un échange de deux courriels entre un médecin qui demande une interprétation des examens qu'il a prescrits à une de ses patientes enceintes et le biologiste qui a les a réalisés. L'enseignant lit le premier courriel et insiste sur le mode interrogatif. II laisse quelques 
secondes aux étudiants puis projette le courriel de réponse qui contient explicitement le message pédagogique.

Treize messages du CM seront délivrés sous une forme neutre, c'est-à-dire par simple lecture et/ou commentaire par l'enseignant des diapositives correspondantes. Pour ces messages qui servent de condition de contrôle, l'enseignant conserve une attitude et une intonation de voix uniques et neutres. Quatre messages sur les dix-sept seront mis en scène (conditions de test): les messages 4 et 12 seront délivrés avec la méthode 1, les messages 8 et 16 le seront avec la méthode 2 (Tableau 1). Le choix de ces messages particuliers repose à la fois sur leur intérêt pédagogique (notions importantes à connaitre) mais aussi sur le fait qu'ils se prêtent plus facilement que d'autres à la mise en scène. L'enseignant devra répéter les mises en scène pour se les approprier. La durée des diapositives varie entre 50 et 90 secondes en fonction du contenu et de la mise en scène.

Tableau 1. Messages pédagogiques du CM (durée totale : 20 minutes). Chaque message est délivré par une diapositive projetée, lue et/ou commentée par l'enseignant. Les messages en gras sont mis en scène $\left({ }^{*}\right.$ méthode $1,{ }^{* *}$ méthode 2 , cf. texte pour plus de détails), les autres messages sont délivrés de manière neutre et servent de condition contrôle.

\begin{tabular}{|c|c|}
\hline Message 1 & $\begin{array}{l}\text { Les modifications physiologiques au cours de la grossesse peuvent } \\
\text { significativement faire varier le bilan sanguin d'une femme enceinte }\end{array}$ \\
\hline Message 2 & Le métabolisme maternel doit s'adapter aux besoins croissants du fœetus \\
\hline Message 3 & Le métabolisme est différent avant et après 20 semaines de grossesse \\
\hline Message $4^{*}$ & Le « yo-yo » glycémique de la femme enceinte \\
\hline Message 5 & $\begin{array}{l}\text { Les paramètres du bilan glycémique sont modifiés au cours de la } \\
\text { grossesse }\end{array}$ \\
\hline Message 6 & Le bilan lipidique est globalement modifié \\
\hline Message 7 & Les variations des lipoprotéines \\
\hline Message $8^{\star *}$ & Le retour à la normale du bilan lipidique après l'accouchement \\
\hline Message 9 & Les besoins fœtaux en protéines \\
\hline Message 10 & Les variations plasmatiques des protéines et acides aminés chez la mère \\
\hline Message 11 & La rétention physiologique de l'eau au cours de la grossesse \\
\hline Message $12^{*}$ & Impacts maternels des besoins du fœtus en calcium et en fer \\
\hline Message 13 & Les besoins maternels en vitamines \\
\hline Message 14 & Les modifications de l'hémoglobine \\
\hline Message 15 & Les modifications de la numération leucocytaire \\
\hline Message $16^{* *}$ & Les modifications de la numération plaquettaire \\
\hline Message 17 & L'état d'hypercoagulabilité sanguine durant la grossesse \\
\hline
\end{tabular}




\subsection{Le recueil des données}

Comme il a été récemment recommandé pour les recherches en neuroscience éducationnelle, (Campbell et Pagé, 2012; Mercier et al., 2012), plusieurs méthodologies seront utilisées pour le recueil des données expérimentales.

\subsubsection{Entretiens et questionnaires}

A l'issue du CM, les étudiants seront interrogés par un intervenant neutre sur leurs opinions et sensations. Ils commenteront de manière générale le cours et citeront les moments qui les ont particulièrement marqués (événements subjectifs). Ce débriefing se fera en revisionnant la vidéo du CM. Pour préciser leur discours, ils répondront ensuite par écrit à dix questions qui portent sur des points spécifiques (l'enseignant, le thème du cours, l'intérêt de certains messages pédagogiques, etc.). Certaines seront ouvertes et d'autres seront à items de Likert. Exemple de questions: $Y$ a-t-il eu durant le cours, un ou plusieurs instants durant lesquels vous avez oublié que vous participiez à une expérience? Réponses possibles (items de Likert): pas d'accord du tout, plutôt pas d'accord, pas d'accord, plutôt d'accord, d'accord, complètement d'accord. Lesquels? (question ouverte). Comment qualifierez-vous la partie sur les besoins en calcium et fer du fotus? (message 12, mis en scène). Comment qualifierez-vous la partie sur les besoins maternels en vitamines? (message 13, neutre). Réponses possibles (items de Likert): très ennuyeux, ennuyeux, plutôt ennuyeux, plutôt intéressant, intéressant, très intéressant. Après synthèse, les réponses concernant les messages neutres et les messages mis en scène seront synthétisées et comparées.

\subsection{2 Électrocardiogramme (ECG)}

Les étudiants porteront durant le cours un enregistreur ECG. Les tracés seront analysés a posteriori. La dynamique cardiaque (dont notamment les intervalles $\mathrm{R}-\mathrm{R}$ ) acquise durant les messages pédagogiques sera comparée à celle des messages neutres.

\subsubsection{Imagerie cérébrale par IRMf}

Une imagerie cérébrale sera obtenue selon le protocole suivant. Le CM sera préalablement enregistré sur vidéo. Avant la session d'IRMf, chaque étudiant rencontrera physiquement l'enseignant. II sera ensuite installé dans le tunnel et équipé d'oreillettes et de lunettes spéciales qui lui permettent d'écouter et de regarder le $\mathrm{CM}$ projeté sur un écran. Brièvement, la comparaison des signaux BOLD (Blood Oxygen Level Dependant) acquis durant les messages 
neutres et les messages mis en scène permettront d'identifier les zones cérébrales spécifiquement activées par la mise en scène pédagogique.

\subsection{4 Évaluation pédagogique}

L'intervention éducative sera évaluée avec un pré-test (juste avant le $C M$ ) et deux post-tests : immédiat (juste après le $\mathrm{CM}$ ) et différé (quinze jours après). Les étudiants répondront à un jeu identique de questions à choix multiples (QCM). Six QCM seront construits, chacun ayant quatre items dont un seul est correct. Chaque QCM se rapporte à un message pédagogique unique et deux QCM sur les six portent sur les messages mis en scène ( $n^{\circ} 4,8,12$ ou 16). La comparaison des réponses permettra d'explorer l'impact éducationnel de la mise en scène des messages et en particulier l'amélioration du processus de mémorisation.

\subsubsection{Les limites de l'étude}

Comme dans toute étude expérimentale, plusieurs limites méthodologiques sont à relever. La première concerne la difficulté de la synchronisation temporelle des différents recueils de données. Les signaux recueillis, notamment pour l'ECG et l'IRMf, devront être associés sans ambiguïté aux messages pédagogiques correspondants. La solution passe par un chronométrage très précis du $\mathrm{CM}$ et des différents enregistrements. La seconde limite est l'obtention d'un ratio signal/bruit suffisant dans les enregistrements et la troisième est la variabilité intra- et interindividuelle des réponses. La conception de l'intervention éducative choisie tente de réduire ces deux difficultés. En effet, deux types différents de messages mis en scène ont été conçus (limiter la variabilité interindividuelle). Quatre messages de ce type ont été dispersés dans le $\mathrm{CM}$ afin de limiter l'effet d'une éventuelle distraction (diminuer la variabilité intra-individuelle). Chaque message mis en scène est encadré par un message neutre afin de créer un effet de contraste (augmenter le rapport signal/bruit). II faut noter ici le rôle important de l'expérimentation à la première personne qui renforce la spécificité des signaux recueillis. Enfin, la dernière limite, et non des moindres, est d'ordre statistique. Ce type d'expérience pilote et interdisciplinaire n'autorise pas les grands effectifs. Si des tests statistiques robustes peuvent prendre en charge des effectifs réduits, l'interprétation des résultats reste problématique. En effet, des résultats non significatifs peuvent provenir d'un manque de puissance, mais des résultats significatifs restent à manier avec prudence. Cette situation constitue un des écueils méthodologiques actuels des neurosciences (Button et al., 2013). 


\section{Les expériences-pilotes}

Pour valider l'hypothèse initiale: "Est-il possible d'induire l'adhésion des étudiants au cours d'une intervention éducative? " deux expériences successives seront menées avec 6 étudiants chacune ( 3 hommes et 3 femmes), répartis par randomisation. Cette répartition permettra de recueillir le maximum de données susceptibles de confirmer l'adhésion des étudiants au $\mathrm{CM}$. Un élément subjectif sera recherché au cours des debriefing des deux expériences: l'oubli transitoire des étudiants qu'ils participent à une expérimentation. Cet oubli qui témoigne de la "déconnexion » de la réalité physique est un argument important en faveur de l'adhésion.

\subsection{Expérience 1: Entretiens + ECG + Évaluation pédagogique}

Au cours de cette expérience, les 6 étudiants suivront ensemble le CM délivré par l'enseignant dans une petite salle de cours. Avant le CM, ils seront équipés des enregistreurs ECG et répondront au questionnaire d'évaluation pré-test. Immédiatement après le $\mathrm{CM}$, ils répondront à l'évaluation post-test. Ils seront ensuite débarrassés de l'enregistreur puis participeront aux entretiens individuels avec des intervenants neutres. Quinze jours après l'expérience, ils recevront de nouveau le questionnaire d'évaluation qu'ils renverront par courriel. Un mois après l'expérience, ils recevront une version écrite intégrale du CM.

L'adhésion des étudiants sera confirmée s'il y a convergence des évènements subjectifs recueillis lors des entretiens et de la variation du rythme cardiaque avec au moins deux messages mis en scène. L'évaluation permettra de démontrer l'impact de la mise en scène sur les performances des étudiants.

\subsection{Expérience 2: IRMf + ECG + Entretiens}

Avant la session IRMf, les 6 étudiants seront équipés avec l'enregistreur ECG puis rencontreront physiquement l'enseignant. Après la session IRMf durant laquelle le CM aura été projeté, ils participeront aux entretiens individuels avec les intervenants neutres. Le débriefing sera réalisé en visionnant de nouveau la vidéo du CM. L'adhésion sera confirmée s'il y a convergence des événements subjectifs et des variations du rythme cardiaque avec au moins deux messages mis en scène. Les zones cérébrales activées seront identifiées et comparées à celles décrites par Metz-Lutz et al. (2010). 


\section{Conclusion}

L'adhésion à une réalité fictionnelle provoque la dissociation entre l'expérience mentale du spectateur et ses perceptions physiques immédiates. Ce phénomène est sous-tendu par des processus physiologiques et cognitifs définis et reste identifiable a posteriori par le sujet lui-même. Par une analyse philosophique et phénoménologique des liens entre le cours magistral et le théâtre, ce concept d'adhésion a pu être étendu au champ pédagogique. Ce travail en pose les bases théoriques et postule que sa survenue relève d'une mise en scène pédagogique. II propose également une approche expérimentale multidisciplinaire spécifique à la neuroéducation. Les expériences conçues ont pour objectif de recueillir plusieurs types de données qui convergent en faveur de l'adhésion des étudiants, mais aussi de montrer que la mise en scène pédagogique a un impact positif sur l'apprentissage des étudiants.

La confirmation que l'adhésion puisse être déclenchée dans le champ pédagogique après celui du théâtre appelle des prolongements théoriques. II devient possible de concevoir que l'adhésion est nécessaire à l'émergence d'une réalité et qu'inversement, il ne peut y avoir émergence d'une réalité sans adhésion. L'adhésion d'un sujet à une représentation, une idée ou un discours constituerait ainsi un méta-phénomène qui ouvre de nouvelles perspectives de recherche en psychologie cognitive. 


\section{Références}

Baruch, Y. (2006). Role-play teaching: acting in the classroom. Management Learning, 37(1), 43-61. https://doi.org/10.1177/1350507606060980

Bressan, Y. (2013). À la recherche du principe d'adhésion : le théâtral comme tube à essais pour les neurosciences cognitives. Paris : L'Harmattan.

Bressan, Y. (2012a). Du principe d'adhésion au théâtre, Approche historique et phénoménologique. Paris : L'Harmattan.

Bressan, Y. (2012b). La « mise en scène pédagogique » : quand la neuroesthétique rencontre la neuro-éducation. Dans A. Yamna et P. Lartigau (dir), Théâtre et éducation. Partage, enjeux, avenirs (p. 100-115). Pessac, France : Presses Universitaires de Bordeaux.

Button, K. S., loannidis, J. P. A., Mokrysz, C., Nosek, B. A., Flint, J., Robinson, E. S. J. et Munafò, M. R. (2013). Power failure: Why small sample size undermines the reliability of neuroscience. Nature Reviews Neurosience, 14(5), 365-376. https://doi.org/10.1038/nrn3475

Campbell, S. R. et Pagé, P. (2012). La neuroscience éducationnelle: enrichir la recherche en éducation par l'ajout de méthodes psychophysiologiques pour mieux comprendre l'apprentissage. Neuroéducation, 1(1), 115-144. https://doi.org/10.24046/neuroed.20120101.115

Charle, C. (1997). La mémoire des lieux. Sociétés contemporaines, 28(1), 103110. https://doi.org/10.3406/socco.1997.1549

Dohn, B. N., Madsen, P. T. et Malte, H. (2009). The situational interest of undergraduate students in zoophysiology. Advances in Physiology Education, 33(3), 196-201. https://doi.org/10.1152/advan.00038.2009

Feagan, R. et Rossiter K. (2011). University-community engagement: A case study using popular theatre. Education + Training, 53(2/3), 140-154. https://doi.org/10.1108/00400911111115681

Gaudé, L. (2000). Onysos le furieux. Arles : Editions Actes-Sud. 
Greenberg, E. et Miller, P. (1991). The player and the professor: Theatrical techniques in teaching. Journal of Management Education, 15(4), 428446. https://doi.org/10.1177/105256299101500405

Griggs, T. (2001). Teaching as acting: considering acting as epistemology and its use in teaching and teacher preparation. Teacher Education Quarterly, 28(2), 23-37. http://www.jstor.org/stable/23478275

Harris, R. J. (1977). The teacher as actor. The Teaching of Psychology, 4(4), 185-187. https://doi.org/10.1207/s15328023top0404 7

Hidi, S. et Renninger, K. A. (2006). The four-phase model of interest development. Educational Psychologist, 41(2), 111-127. https://doi.org/10.1207/s15326985ep4102 4

Macherey, P. (2011). Le cours magistral (2). En ligne : http://philolarge.hypotheses.org/1026

Mercier, J., Léger, P.-M., Girard, C. et Dion, J.-S. (2012). Bridging the gap between cognitive neuroscience and education: Psychophysiological and behavioral data collection in authentic contexts. Neuroeducation, 1(1), 5-28. https://doi.org/10.24046/neuroed.20120101.5

Metz-Lutz, M. N., Bressan, Y., Heider, N. et Otzenberg, H. (2010). What physiological changes and cerebral traces tell us about adhesion to fiction during theater-watching? Frontiers in Human Sciences, 4(59), 110. https://doi.org/10.3389/fnhum.2010.00059

Murray, H. G. et Lawrence, C. (1980). Speech and drama training for lectures as a means of improving university teaching. Research in Higher Education, 13(1), 73-90. https://doi.org/10.1007/bf00975777

Noguès, B. (2008). Élèves ou auditeurs? Le public des facultés de lettres et sciences au. XIX ${ }^{e}$ siècle (1808-1878). Histoire de l'Education, (120), 7797. https://doi.org/10.4000/histoire-education.1834

Pineau, E. L. (1994). Teaching is performance: Reconceptualizing a problematic metaphor. American Educational Research Journal, 31(1), 3-25. https://doi.org/10.3102/00028312031001003 
Schraw, G. et Lehman, S. (2001). Situational interest: A review of the literature and directions for future research. Educational Psychology Review, 13(1), 23-52. https://doi.org/10.1023/A:1009004801455

Souto-Manning, M. (2011). Playing with power and privilege: Theatre games in teacher education. Teaching and Teacher Education, 27(6), 997-1007. https://doi.org/10.1016/j.tate.2011.04.005

Waack, W. L. (1983) Teaching and acting: the conconant arts. Teacher Education Quaterly, 10(2), 72-87. http://www.jstor.org/stable/23474397 